\title{
Seasonal Distribution and Meteorological Factors Associated with Hand, Foot, and Mouth Disease among Children in Xi'an, Northwestern China
}

\author{
Tianci Guo, ${ }^{1}$ Jifeng Liu, ${ }^{2}$ Junjiang Chen, ${ }^{1}$ Yao Bai, ${ }^{1,2}$ Yong Long, ${ }^{1}$ Baozhong Chen, ${ }^{2}$ Shuxuan Song,,${ }^{1}$ Zhongjun Shao, ${ }^{1 \star}$ \\ and Kun Liü* \\ ${ }^{1}$ Department of Epidemiology, Ministry of Education Key Lab of Hazard Assessment and Control in Special Operational Environment, School \\ of Public Health, Air Force Medical University, Xi'an, P. R. China; ' ${ }^{2}$ Department of Infectious Disease Control and Prevention, Xi'an Center for \\ Disease Prevention and Control, Xi'an, P. R. China
}

\begin{abstract}
Hand, foot, and mouth disease (HFMD) is a common infectious disease in the Asia-Pacific region that primarily affects children younger than 5 years. Previous studies have confirmed that the seasonal transmission of this disease is strongly related to meteorological factors, but the results are not consistent. In addition, the associations between weather conditions and HFMD in northwestern China have not been investigated. Therefore, we aimed to examine this issue in Xi'an, the largest city of northwestern China that has been suffering from serious HFMD epidemics. In the current study, data for HFMD and six meteorological factors were collected from 2009 to 2018 . Using crosscorrelation analysis, the Granger causality test, and the distributed lag nonlinear model, we estimated the quantitative relationships and exposure-lag-response effects between weekly meteorological factors and HFMD incidence among children. We found that the seasonal distribution of HFMD in Xi'an has two peaks each year and is significantly impacted by the weekly temperature, precipitation, and evaporation over an 8-week period. Higher values of temperature and evaporation had positive associations with disease transmission, whereas the association between precipitation and HFMD showed an inverted- $U$ shape. The maximum relative risks (RRs) of HFMD for the weekly mean temperature (approximately $\left.31.1^{\circ} \mathrm{C}\right)$, weekly cumulative evaporation $(57.9 \mathrm{~mm})$, and weekly cumulative precipitation $(30.0 \mathrm{~mm})$ were 1.56 (95\% Cl: $1.35-1.81), 1.40$ (95\% Cl: 1.05-1.88), and 1.16 (95\% Cl: 1.11-1.70), respectively. The identified risk determinants and lag effects could provide important information for early interventions to reduce the local disease burden.
\end{abstract}

\section{INTRODUCTION}

Hand, foot, and mouth disease (HFMD) is a common infectious disease caused by enteroviruses (e.g., EV-71, Coxsackievirus 16 [CV-A16], CV-A4, CV-A6, and CV-A10) that primarily affects children younger than 5 years. ${ }^{1} \mathrm{Al}-$ though the symptoms are usually mild (fever, skin rash on the hands and feet, and vesicles in the mouth), some rare complications can lead to cognitive and motor disorders as well as death. ${ }^{2,3}$ Therefore, this disease poses a serious threat to public health in countries with a high incidence, such as China and other Asia-Pacific countries. ${ }^{4-8}$ China accounted for $86 \%$ of the total cases reported to the WHO between 2010 and 2014, and it reported an alarming number of approximately 2.7 million cases of HFMD, resulting in 384 deaths in $2014 .{ }^{9}$ Although vaccines against EV-71 for children aged 6 months to 5 years have been available since 2015, no effective vaccines for other Coxsackieviruses exist yet, and the incidence of HFMD has not decreased. ${ }^{10} \mathrm{Be}-$ cause HFMD is a major public health issue in China, ranking first among all notifiable infectious diseases and affecting more than two million children annually, it is of utmost importance to identify the risk factors of HFMD for better prevention and control of the disease. ${ }^{4,11,12}$

Some known risk factors for HFMD include hygiene, age, gender, and social contacts. ${ }^{1}$ In addition, HFMD is a climatesensitive disease, and epidemics of HFMD have commonly shown seasonal variations and geographical differences. ${ }^{1,4}$

*Address correspondence to Zhongjun Shao or Kun Liu, Department of Epidemiology, Ministry of Education Key Lab of Hazard Assessment and Control in Special Operational Environment, School of Public Health, Air Force Medical University, 169 West Changle Rd., Xincheng District, Xi'an 710032, P. R. China. E-mails: 13759981783@ 163.com or liukun5959@qq.com
For example, HFMD has shown two peaks in May and October in southern China, one peak in June in northern China, and one peak in March or May in Singapore. ${ }^{4,6}$ Meteorological factors, such as temperature, relative humidity, precipitation, hours of sunshine, and air pressure, also have been shown to play important roles in transmission of this disease. ${ }^{13,14}$ However, associations between meteorological factors and HFMD have not been consistent in previous studies. ${ }^{6,8,13,15,16}$ A linear association has been reported between temperature and HFMD in Guangzhou (southern China), ${ }^{13}$ whereas studies from Shanghai, Singapore, and Japan have revealed nonlinear associations. 6,8,15 Moreover, some meteorological factors, such as relative humidity and temperature, have shown opposite impacts on the transmission of HFMD in different regions. ${ }^{13,16}$ It has been hypothesized that these heterogeneous findings among studies are due to differences in types of data, modeling schemes, and, most importantly, region-specific characteristics, such as climatic, socioeconomic, demographic, and infrastructural conditions. ${ }^{15,17}$ Although a few studies have been conducted in China (Being, Shanghai, Guangzhou, Chengdu, and Hefei), ${ }^{13,16,18-20}$ to the best of our knowledge, no study has examined the association between meteorological factors and HFMD in northwestern China. This information is of utmost importance to design effective and targeted interventions to reduce the transmission of HFMD, according to local conditions.

Therefore, the aim of this study was to assess the association between weather conditions and HFMD in Xi'an, the largest city in northwestern China with serious HFMD epidemics. Specifically, we aimed to 1) explore the incidence of HFMD in Xi'an; 2) assess the association between epidemiological characteristics, especially the seasonal distribution, and incidence of HFMD; and 3) evaluate the exposure-lagresponse effects of meteorological factors on the incidence of HFMD. 


\section{MATERIALS AND METHODS}

Study area. Xi'an lies on the Wei River Basin, a flood plain created by eight surrounding rivers and streams in the central part of Shaanxi Province with a high-density population, where it is suitable for the spread of HFMD. From 2008 to 2015 , a total of 154,869 HFMD cases were reported in Xi'an city, with an annual incidence of 235.01 per 100,000 people, which is nearly two times the national average incidence during the same period. ${ }^{21}$ Xi'an consists of 13 counties and districts with an administrative area of $9,983 \mathrm{~km}^{2}$, and the total population was more than 10 million residents in 2018 (Supplemental Figure 1). The city has an average elevation of $400 \mathrm{~m}$ above sea level and a typical temperate continental monsoon climate, with pleasant temperatures, moderate rainfall, and four distinct seasons: hot and wet in the summer, dry and seldom snowy in the winter, and prone to extended periods of rain in the spring and autumn. January is the coldest month, with an average temperature of $-1.3^{\circ} \mathrm{C}$; and July is the hottest month, with an average temperature of $26.7^{\circ} \mathrm{C}$. The average annual rainfall is $604.2 \mathrm{~mm}$, which is concentrated in July, August, and September (available from https://www.tianqi.com/xian/).

Data source. Case records of HFMD in Xi'an city from January 1, 2009 to December 31, 2018 were obtained from the Xi'an Center for CDC. We included all cases, without any exclusion criteria. In China, all clinics are obliged to report HFMD cases to the local CDC within 24 hours. ${ }^{22}$ The HFMD cases were diagnosed according to the guidelines provided by the National Health and Family Planning Commission of the People's Republic of China (version 2008). ${ }^{23}$ Information regarding gender, age, onset date of symptoms, and residential address was collected for each case from the case records. The annual demographic data during the study period were obtained from the Xi'an Bureau of Statistics. A digital township-level map of Xi'an city was gathered from the China Resource and Environment Data Cloud Platform (http://www.resdc.cn/). Data on six local meteorological variables-temperature, precipitation, evaporation, atmospheric pressure, relative humidity, and sunshine duration-were collected daily during the study period from the Chinese Bureau of Meteorology (http://data.cma.cn/).

Statistical analysis. To display the epidemiological characteristics of HFMD in Xi'an in a clear manner, a bar chart of annual HFMD cases and average age- and genderspecific incidences was created for the study period, and a spatial distribution map of the annual HFMD incidence was produced at the township level. Because HFMD cases involving children younger than 5 years accounted for most of the total cases, we counted the weekly number of reported HFMD cases in children younger than 5 years and calculated the weekly incidence in Xi'an, which was expressed by the seasonal distribution of meteorological factors and HFMD incidence using time series plots. Then, we used Spearman's correlation analysis to calculate preliminary correlations among meteorological factors and the HFMD incidence. In addition, a Granger causality test was performed for each meteorological factor to determine the potential effect of meteorological variability on the transmission of HFMD. Subsequently, the variables selected in the Granger causality test were included in a distributed lag nonlinear model (DLNM) to examine the potentially nonlinear and delayed effects of meteorological factors on disease transmission. The DLNM is based on the definition of crossbasis, a bidimensional function expressed as the combination of two basic functions, which depicts the effects of predictor and lag simultaneously. Two functions, "crossbasis" and "crosspred," are included in the DLNM and are used to study the lag effect and prediction, respectively, as well as to estimate the exposure-response prediction across the maximum lag period. ${ }^{24}$ In the current study, we used the DLNM combined with the generalized additive model to analyze the effects. A number of covariates were incorporated through the Poisson regression model, as follows:

$$
\begin{aligned}
\mathrm{Yt} \sim \operatorname{Poisson}(\mu)=\alpha & +\mathrm{NS}(\beta, \mathrm{df}, \text { lag, } \mathrm{df}) \\
& +\mathrm{NS}(\text { time, } \mathrm{df})+\text { week }
\end{aligned}
$$

where Yt is the incidence of HFMD on week $t$, $\alpha$ is the intercept, NS is a natural cubic spline used to model the nonlinear relationship between the meteorological variables and the incidence, $\beta$ is the examined meteorological variable that was strongly related to the incidence, $\mathrm{df}$ is the degrees of freedom of each meteorological variable per year, time is the indicator variable used to control long-term trends and seasonality, and week is an ordinal variable for the week of each year.

The analysis was performed using $R$ software version 3.6.1 with the "Imtest" and "dlnm" packages. All statistical tests were two sided, and a $P$-value $<0.05$ was considered statistically significant.

\section{RESULTS}

Epidemiological characteristics of HFMD and meteorological factors. From January 1, 2009 to December 31, 2018 , a total of 181,358 cases and 18 deaths due to HFMD were reported in Xi'an. The ages of the patients ranged from 1 day to 78 years (median: 2 years), and the mean (SD) age was 2.55 (2.51) years. Children younger than 5 years with HFMD accounted for $94.5 \%(162,152 / 181,358)$ of the total cases. The ratio of males to females of all cases was $1.43: 1$, and males had a significantly higher incidence than females among all age-groups $(P<0.001)$. The highest annual agespecific incidence occurred between 1 and 4 years of age, with an incidence greater than $5,000 / 100,000$ people (Figure 1). The high epidemic region of HFMD mainly occurred at the junctions of urban-rural zones around Xi'an (Supplemental Figure 2). Details of the weekly meteorological factors and HFMD incidence during the study period are shown in Table 1, Figure 2. Specifically, a weekly time-series diagram of HFMD incidence in children younger than 5 years and meteorological factors indicated significant seasonal variation (Figure 2). The HFMD incidence had a slow increasing trend, showing an obvious bimodal curve with a large peak in late spring and early summer from the 17th week to the 29th week of each year, and a small peak in late autumn and early winter between the 38th week and the 49th week (Figure 2). The weekly values for average temperature, accumulative precipitation, accumulative evaporation, average atmospheric pressure, average relative humidity, and accumulative sunshine duration are shown in Table 1.

The cross-correlation analysis revealed that three meteorological factors-temperature, precipitation, and evaporation-were 


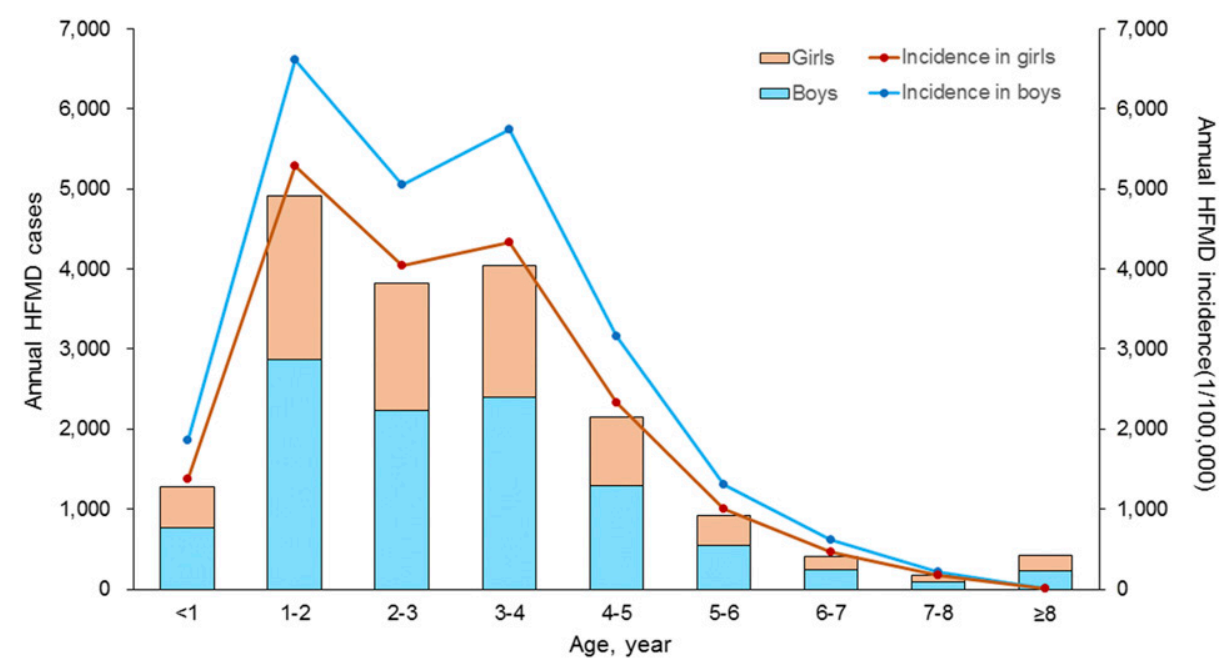

FIGURE 1. Age and gender distribution of hand, foot, and mouth disease cases in Xi'an city, northwestern China, 2009-2018. This figure appears in color at www.ajtmh.org.

positively correlated with the HFMD incidence, and atmospheric pressure was negatively associated with the disease (Figure 3). The results of the Granger causality tests indicated that the temporal distribution of weekly HFMD incidence was significantly affected by temperature, precipitation, and evaporation $(P<0.05)$ (Table 2).

Nonlinear and lag effects of meteorological factors on the HFMD incidence. Figure 4A shows the comprehensive association between the average weekly temperature and the incidence of HFMD along with the lag time (0-8 weeks) (also shown in Supplemental Figure 3). Within 2 weeks, the relative risk (RR) of HFMD increased significantly as the temperature increased, but after 2 weeks, the impact of temperature became negative. The separate effects on the RRs together with the $95 \% \mathrm{Cl}$ are shown in Figure 4B. The RR of HFMD with temperature was positive at a lag time of 0 , and it decreased substantially as the lag time increased. The maximum RR was $1.56(95 \% \mathrm{Cl}: 1.35-1.81)$ at approximately $31.1^{\circ} \mathrm{C}$ in the current week (lag time of 0 ) (Table 3 ). The effect of temperature on the cumulative risk of HFMD is shown in Figure 4C. A higher temperature increased the cumulative risk from a lag time of 0 to 2 weeks; and the highest RR value of 2.27 (95\% Cl: 1.59-3.23) appeared at approximately $31.1^{\circ} \mathrm{C}$, with a lag time of 2 weeks.

The association between weekly precipitation and HFMD showed an inverted-U shape during the 8-week period following the measurement. With an increase of precipitation, the $\mathrm{RR}$ of HFMD rose to the peak at about $30 \mathrm{~mm}$ of precipitation and then declined rapidly (Figure 5A, Supplemental Figure 4). With a weekly precipitation of $20-40 \mathrm{~mm}$, the separate and cumulative effects were positive (Figure 5B and C). The separate effects were the strongest at about $30.0 \mathrm{~mm}$ of precipitation with a lag time of 0 weeks, with a maximum $R R$ value of 1.16 (95\% Cl: 1.11-1.70) (Table 3); and the largest cumulative effects occurred at $24.9 \mathrm{~mm}$ of precipitation with a lag time of 7 weeks, with a RR value of 2.03 (95\% Cl: 1.73-2.37) (Table 3).

Figure 6A shows the combined effect of lag time and evaporation on the RR of HFMD. Given the high correlation between evaporation and temperature $(r=0.80)$, the effects of weekly evaporation on HFMD were similar to those of temperature; however, the effects of evaporation lasted a longer time (5 weeks), with higher values between $30 \mathrm{~mm}$ and $40 \mathrm{~mm}$ of evaporation (Supplemental Figure 5). The separate RR increased with a rise of evaporation, and the coefficient of variation increased simultaneously. The RR of weekly evaporation of $57.9 \mathrm{~mm}$ with a lag time of 0 week was the highest, with a value of 1.40 (95\% Cl: 1.05-1.88) (Figure 6B, Table 3). The largest cumulative $\mathrm{RR}$ value of 2.40 (95\% Cl: $1.66-3.46)$ appeared at an evaporation of approximately $41.7 \mathrm{~mm}$, with a lag time of 5 weeks (Figure 6C, Table 3).

\section{DISCUSSION}

In the current study, a total of 181,358 HFMD cases were reported in Xi'an city, with an annual incidence of 213.88 per

TABLE 1

Descriptive statistics of weekly meteorological factors and HFMD incidence among children younger than 5 years in Xi'an, northwestern China, 2009-2018

\begin{tabular}{|c|c|c|c|c|c|c|}
\hline Variable & Min & $P_{25}$ & $P_{50}$ & $P_{75}$ & Max & Mean \pm SD \\
\hline Temperature $\left({ }^{\circ} \mathrm{C}\right)$ & -5.75 & 3.99 & 13.94 & 21.10 & 31.13 & $12.94 \pm 9.58$ \\
\hline Precipitation (mm) & 0 & 0.52 & 6.16 & 18.40 & 111.79 & $13.58 \pm 19.00$ \\
\hline Evaporation (mm) & 1.54 & 11.92 & 20.01 & 29.84 & 64.09 & $21.89 \pm 11.88$ \\
\hline Atmospheric pressure $(\mathrm{hPa})$ & 918.82 & 929.54 & 936.31 & 942.35 & 963.28 & $936.63 \pm 9.41$ \\
\hline Relative humidity (\%) & 28.31 & 55.28 & 65.72 & 74.82 & 93.29 & $65.06 \pm 13.02$ \\
\hline Sunshine duration $(\mathrm{h})$ & 0.23 & 24.89 & 37.40 & 49.00 & 82.54 & $37.33 \pm 16.24$ \\
\hline HFMD incidence (1/100,000 people) & 0.74 & 17.94 & 48.13 & 109.44 & 460.45 & $82.08 \pm 93.84$ \\
\hline
\end{tabular}

$\mathrm{HFMD}=$ hand, foot, and mouth disease; Max $=$ the maximum level of the variable; Min = minimum level of the variable; $P_{25}=$ the 25 th percentile of the variable; $P_{50}=$ the 50 th percentile of the variable; $\mathrm{P}_{75}=$ the 75 th percentile of the variable. 


\section{Weekly meteorological factors and HFMD incidence in Xi'an}

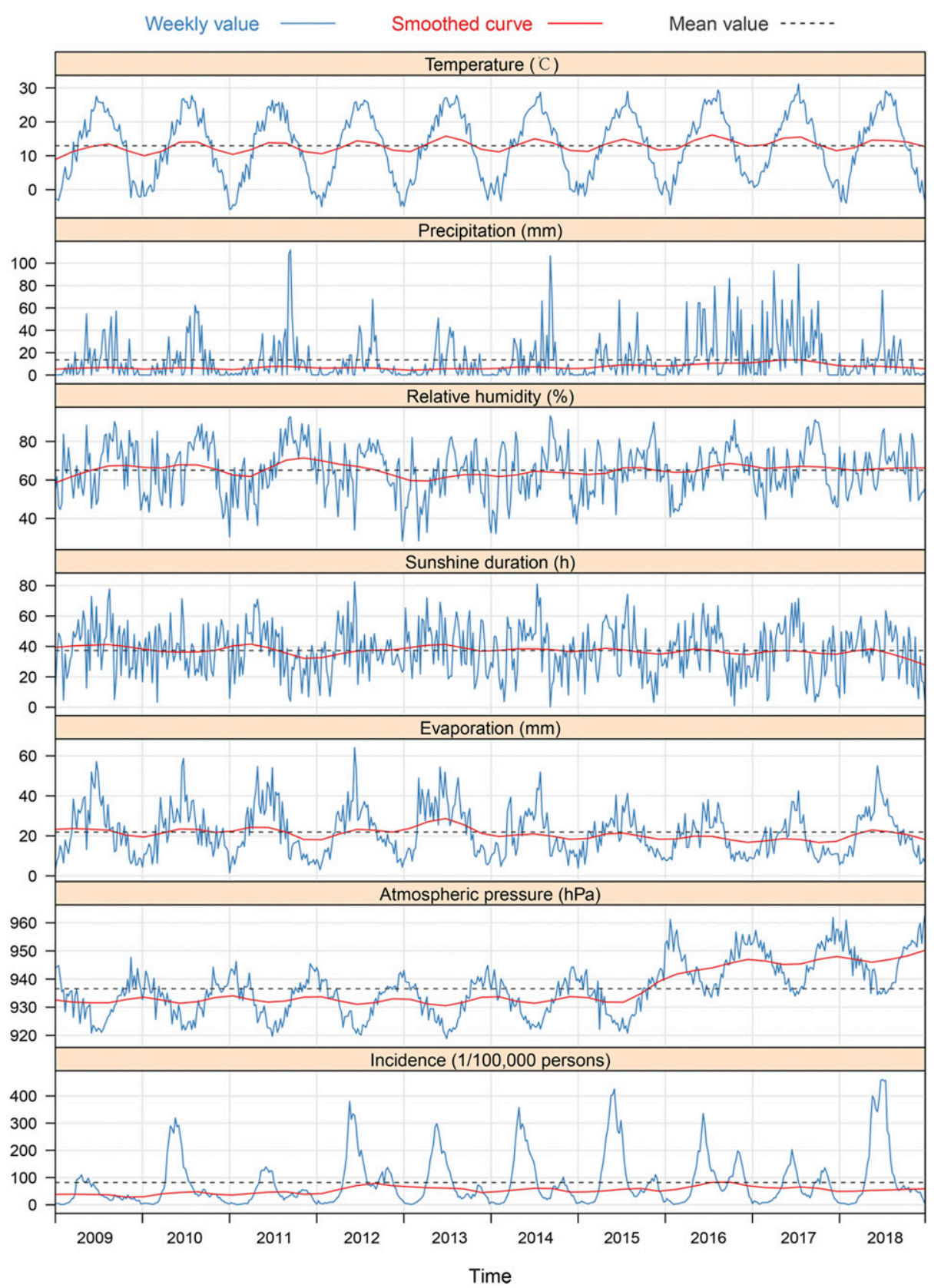

FIGURE 2. Weekly incidence of hand, foot, and mouth disease (HFMD) and meteorological variables in Xi'an city, northwestern China, 2009-2018. (From top to bottom, the curves are temperature, precipitation, relative humidity, sunshine duration, evaporation, atmospheric pressure, and incidence of HFMD, respectively). This figure appears in color at www.ajtmh.org.

100,000 people, ranking first among all notifiable infectious diseases from 2009 to 2018 . We found that children younger than 5 years accounted for $94.5 \%$ of the HFMD cases, and males had a higher incidence than females. Our results demonstrated that the weekly meteorological variables, especially temperature, precipitation, and evaporation, contributed significantly to the seasonal fluctuations of HFMD incidence among children. Significantly, we observed nonlinear lag effects in the relationships between short-term weather conditions and HFMD, thus providing valuable evidence for decisions regarding local public health. To the best of our knowledge, this is the first study to estimate the seasonal distribution and risk determinants of HFMD among children in Xi'an, northwestern China, in a systematic manner.

The incidence of HFMD observed in the current study was significantly higher than the average national incidence as well as that for other large cities in China (such as Beijing, Wuhan, Taiyuan, Shenyang, and Tianjin). ${ }^{21,25-28}$ The results of previous studies are consistent with the current finding that children younger than 5 years account for most of the HFMD cases, and males have a higher incidence than females. ${ }^{29}$ However, prior studies have found different seasonal patterns 


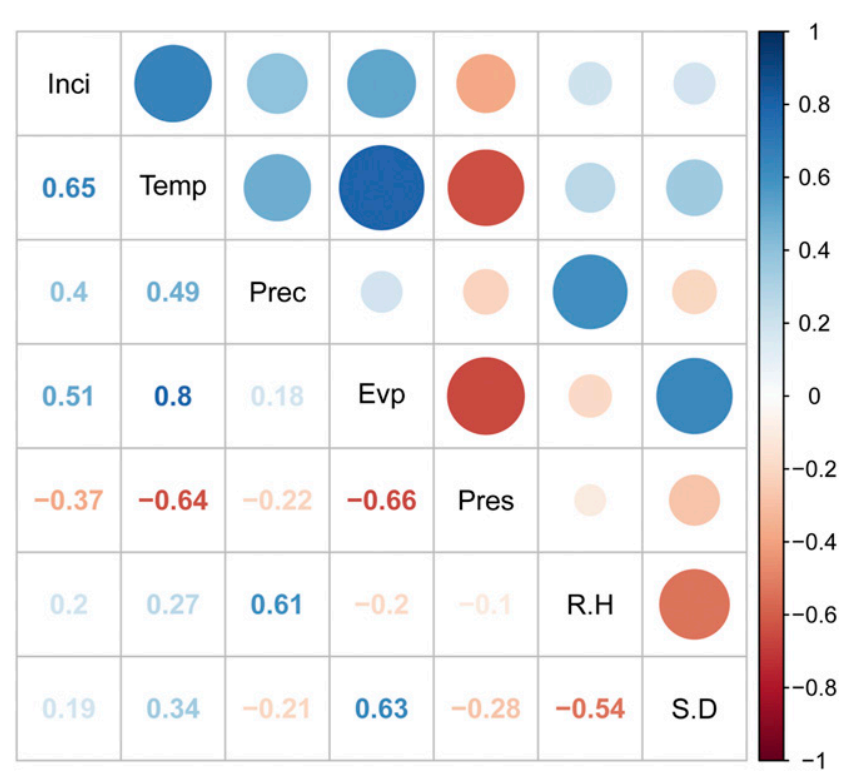

FIGURE 3. Cross-correlation coefficients between the weekly meteorological variables and the incidence of hand, foot, and mouth disease (HFMD) in Xi'an city, northwestern China, 2009-2018 (Inci: weekly incidence of HFMD; Temp: weekly mean temperature; Prec: weekly cumulative precipitation; Evp: weekly cumulative evaporation; Pres: weekly mean atmospheric pressure; R.H: weekly mean relative humidity; S.D: weekly cumulative sunshine duration). This figure appears in color at www.ajtmh.org.

of HFMD compared with the current study. In the present study, we found a large peak for HFMD incidence in late spring and early summer as well as a small peak in late autumn and early winter in Xi'an; this finding was different from those observed in northern China, which only showed one peak in June. ${ }^{4}$ The observed heterogeneity supported the hypothesis that regionspecific characteristics could explain the discrepant findings among studies and reinforced the importance of investigating the region-specific epidemiology of HFMD for more tailored intervention and prevention policies. ${ }^{15,17}$

In addition, the current study used the DLNM to describe the potentially nonlinear and lag effects in the time-series data; this model has been widely used to estimate the relationships between seasonal variations of infectious diseases, such as malaria, HFMD, tuberculosis, and mumps, and meteorological factors. ${ }^{15,16,19,30-32}$ As a result, we found that meteorological factors, including temperature, precipitation, and evaporation, had a significant impact on the occurrence of HFMD, with a lag period of 0 to 8 weeks. The positive role that temperature plays in the incidence of HFMD is well understood. The increased temperature may lead to enhanced survival, reproduction, and transmission of enteroviruses as well as higher chances of exposure for children through contact. ${ }^{33-35}$ An increased temperature also has been shown to lower immunity in humans as well as to facilitate the spread and susceptibility of
HFMD among children. ${ }^{36-38}$ In the present study, we observed a positive impact of temperature within a short time of 2 weeks, after which the impact became negative. This may be due to a rapid change in temperature, which may cause people to alter their activities accordingly. Evaporation has been observed to have a high correlation with temperature; therefore, not surprisingly, the effects of evaporation on HFMD were similar to those of temperature. Furthermore, higher levels of evaporation may increase the transmission of virus through the additional promotion of air flow. ${ }^{39}$ Global climate change may be a primary contributor to the change in temperature, and children represent the most vulnerable group and are particularly at greater risk of disease. ${ }^{40}$

Previous studies have found a negative linear relationship between monthly precipitation and HFMD in eastern central China and Vietnam, which is different from the inverted-U pattern relationship observed in Xi'an. ${ }^{41-44}$ The positive association between precipitation and HFMD may be due to the moisture content in the surface soil or water, which mimics the water content in human feces, thus enabling and activating the virus and affecting the activity and transmission of the enteroviruses so that they are more contagious and survive longer. ${ }^{45,46}$ On the other hand, the negative association between precipitation and HFMD could be explained by the fact that because of excessive precipitation, children spend less time participating in outdoor activities and have a decreased risk of coming into contact with enteroviruses. Thus, the incidence of HFMD decreases with increasing precipitation.

Over the past few decades, HFMD has emerged as a serious public health threat worldwide because of its high incidence and severe complications. ${ }^{1}$ Although the incidence of HFMD in Xi'an is high and has shown a slowly increasing trend, the case fatality rate (CFR) of this disease has declined and is significantly lower than the national average level. ${ }^{47}$ The increased incidence and decreased CFR may reflect the improvements in disease reporting and surveillance, suggesting that HFMD cases have been reported in a timely and accurate manner, subsequently leading to timely and effective treatment. Although the incidence of HFMD in Xi'an has not decreased after the widespread use of the EV-71 vaccine since 2017, the number of severe HFMD cases due to EV-71 infection has decreased dramatically, which is a good sign. However, high and inapparent infection rates of HFMD have been observed mainly among children younger than 4 years, peaking between April and June in Xi'an, suggesting that preventative measures should be installed for high-risk children in addition to vaccination before the epidemic seasons every year. ${ }^{48}$

The results from the current study have important public health implications, such as meteorological factors, namely, weekly temperature, precipitation, and evaporation, could be considered as useful references in the early warning system for the disease transmission. In Xi'an, high temperature and evaporation days (weekly temperature higher than $20^{\circ} \mathrm{C}$ and weekly evaporation higher than $30 \mathrm{~mm}$ ), and moderate

TABLE 2

Granger causality tests for each meteorological variable and the weekly incidence of hand, foot, and mouth disease in Xi'an city, northwestern China, 2009-2018

\begin{tabular}{lcccccc}
\hline & Temperature & Precipitation & Relative humidity & Sunshine duration & Evaporation & Atmospheric pressure \\
\hline F-statistic & 3.808 & 1.996 & 1.353 & 1.297 & 2.469 & 1.041 \\
$P$-value & $<0.001$ & 0.0451 & 0.215 & 0.257 & 0.012 & 0.404 \\
\hline
\end{tabular}


A

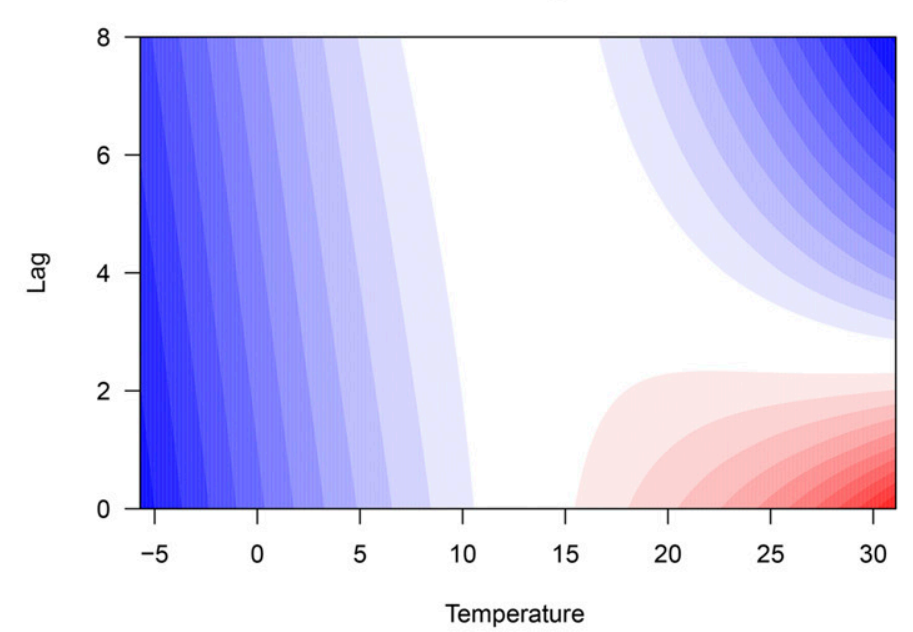

RR

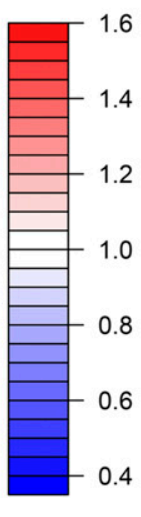

B
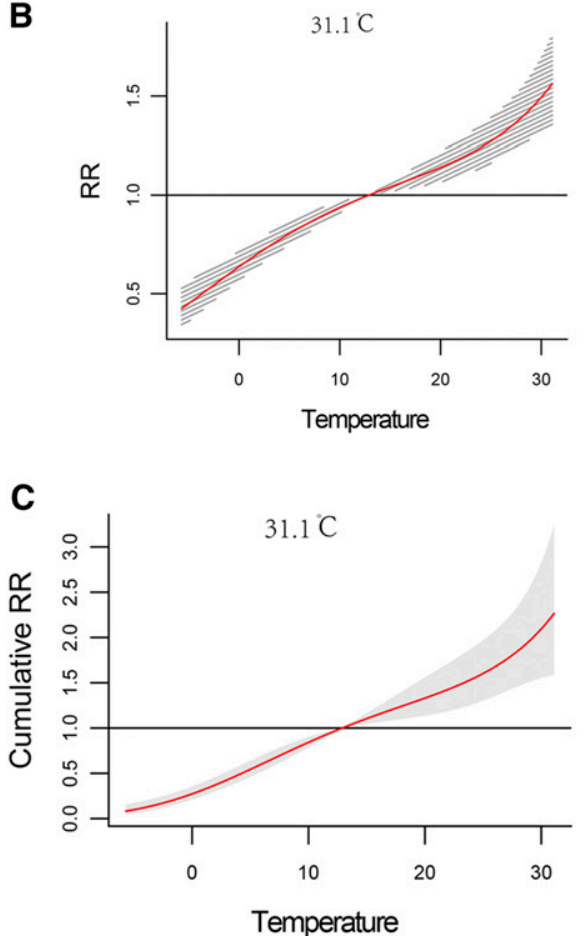
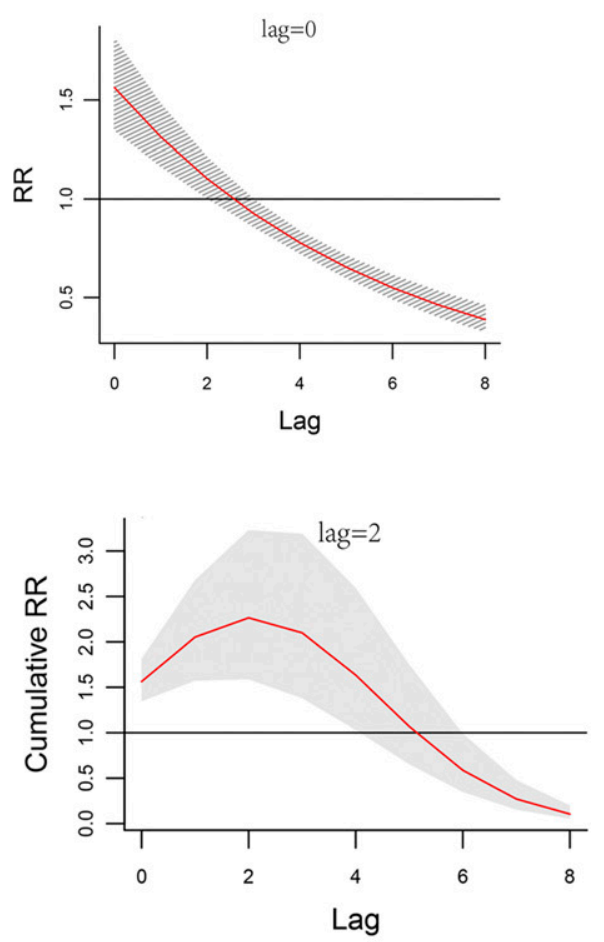

FIGURE 4. Nonlinear and lag effects of temperature on the incidence of hand, foot, and mouth disease (HFMD) in Xi'an city, northwestern China, 2009-2018. (A) Contour plots of the combined effect of lag time (weeks) and temperature on the relative risk (RR) of HFMD transmission. (B) Effects of specific temperature and lag time (weeks) on the RR of HFMD transmission. The red lines indicate the mean RRs, and the gray lines indicate the $95 \% \mathrm{Cl}$. (C) Effect of specific temperature and lag time (weeks) on the cumulative risk of HFMD transmission. The red lines indicate the mean cumulative risks, and the gray areas correspond to the $95 \%$ Cls. This figure appears in color at www.ajtmh.org.

precipitation days (weekly precipitation of 20-40 $\mathrm{mm}$ ) would significantly raise the risk of HFMD. Therefore, targeted strategies and measures to prevent and control epidemics should be conducted in this period, such as rigorous disinfection measures in the kindergartens and playgrounds, and public health education regarding combating HFMD could be enhanced, through TV, radio, or posters, to increase the awareness of HFMD among people.

TABLE 3

Separate and cumulative effects of meteorological factors on the weekly hand, foot, and mouth disease incidence and the corresponding variable values in Xi'an city, northwestern China, 2009-2018

\begin{tabular}{|c|c|c|c|c|c|c|}
\hline \multirow[b]{2}{*}{ Variable } & \multicolumn{3}{|c|}{ Separate effect } & \multicolumn{3}{|c|}{ Cumulative effect } \\
\hline & Maximum RR (95\% Cl) & Variable value & Lag time (weeks) & Maximum RR (95\% Cl) & Variable value & Lag time (weeks) \\
\hline Temperature $\left({ }^{\circ} \mathrm{C}\right)$ & 1.56 (1.35-1.81) & 31.1 & 0 & 2.27 (1.59-3.23) & 31.1 & 2 \\
\hline Precipitation (mm) & 1.16 (1.11-1.70) & 30.0 & 0 & 2.03 (1.73-2.37) & 24.9 & 7 \\
\hline Evaporation (mm) & 1.40 (1.05-1.88) & 57.9 & 0 & $2.40(1.66-3.46)$ & 41.7 & 5 \\
\hline
\end{tabular}

\footnotetext{
$\mathrm{RR}=$ relative risk.
} 
A
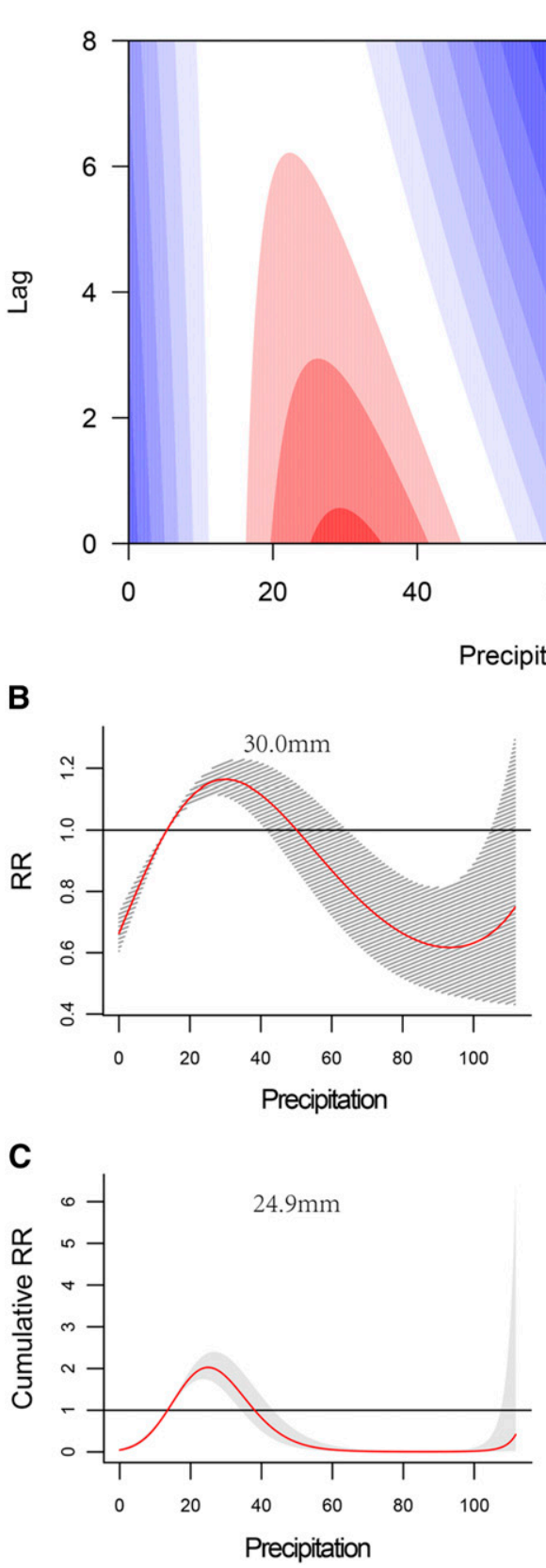

RR

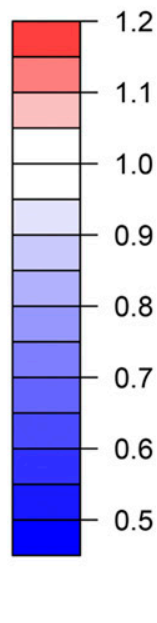

$80 \quad 100$
1

1.0

0.9

0.8

0.7

0.6

0.5
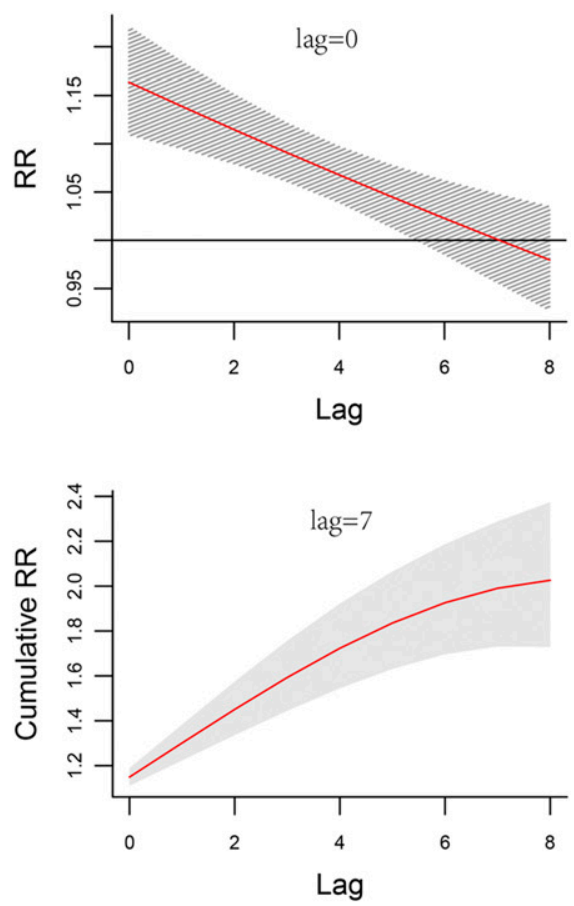

FIGURE 5. Nonlinear and lag effects of precipitation on the incidence of hand, foot, and mouth disease (HFMD) in Xi'an city, northwestern China, 2009-2018. (A) Contour plots of the combined effect of lag time (weeks) and precipitation on the relative risk (RR) of HFMD transmission. (B) Effects of specific precipitation and lag time (weeks) on the RR of HFMD transmission. The red lines indicate the mean RRs, and the gray lines indicate the $95 \%$ Cls. (C) Effects of specific precipitation and lag time (weeks) on the cumulative risk of HFMD transmission. The red lines indicate the mean cumulative risks, and the gray areas indicate the $95 \%$ Cls. This figure appears in color at www.ajtmh.org

Several limitations merit consideration. First, we used data from a hospital-based passive surveillance system, which only captured patients with HFMD who sought medical care, and did not include all cases. Therefore, the prevalence of HFMD in the current study may be underestimated. Second, some information was not collected in the hospital system, such as the types of pathogens responsible for the HFMD cases; thus, we were unable to investigate the specific impacts of meteorological factors on different pathogens, which may have different tolerances to meteorological factors, such as temperature and humidity. In addition, we could not explore the residual confounding from demographic, socioeconomic, behavioral, and physiological factors that may explain the associations between meteorological variables and HFMD incidence. Although the current data source was from the governmental sector and is likely to be highly credible, data were not available to demonstrate that the accuracy of the reporting system is the same in all areas in this study. Future studies with a prospective study design and comprehensive data collection in Xi'an and northwestern China are warranted to validate our findings and address the aforementioned issues. 
A

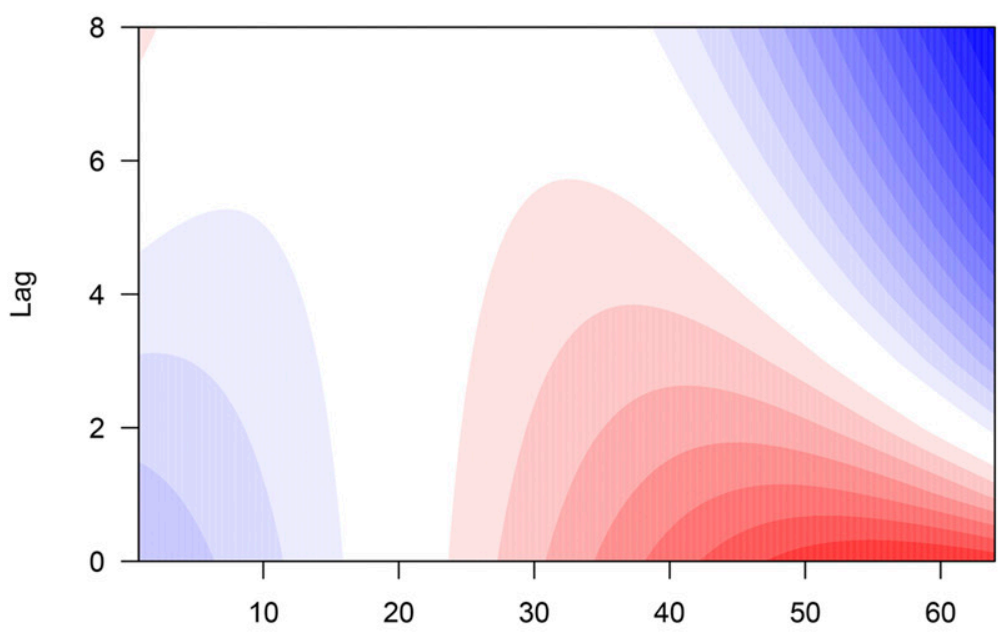

RR

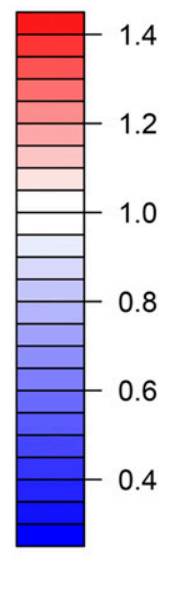

Evaporation

B
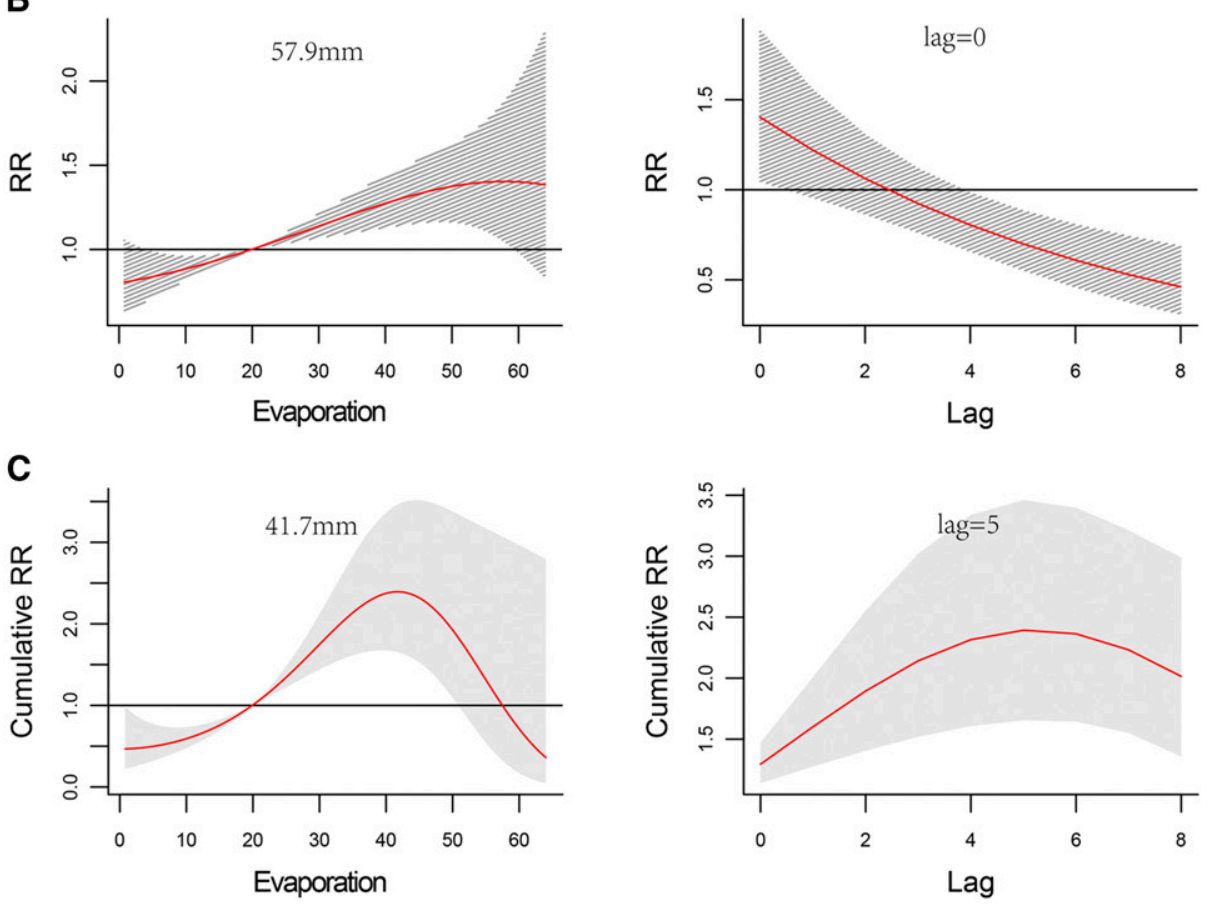

FIGURE 6. Nonlinear and lag effects of evaporation on the incidence of hand, foot, and mouth disease (HFMD) in Xi'an City, northwestern China, 2009-2018. (A) Contour plots of the combined effect of evaporation and lag time (weeks) on the relative risk (RR) of HFMD transmission. (B) Effects of specific evaporation and lag time (weeks) on the RR of HFMD transmission. The red lines indicate the mean RRs, and the gray lines indicate the $95 \%$ Cls. (C) Effect of specific evaporation and lag time (weeks) on the cumulative risk of HFMD transmission. The red lines indicate the mean cumulative risks, and the gray areas indicate $95 \%$ Cls. This figure appears in color at www.ajtmh.org

\section{CONCLUSION}

Our study showed significant associations between meteorological factors (temperature, precipitation, and evaporation) and seasonal variation of HFMD in Xi'an, China. The key determinants of HFMD transmission and the identified lag effects in the current study provide significant evidence and useful references for early warning and the development of targeted strategies and measures to prevent and control local epidemics more effectively.
Received December 9, 2019. Accepted for publication February 1, 2020.

Published online March 9, 2020.

Note: Supplemental figures appear at www.ajtmh.org.

Acknowledgments: This work was supported by the National Natural Science Foundation of China (81803289 and 81773488), a China Special Grant for the Prevention and Control of Infectious Diseases (2017ZX10105011), and the Military Medicine Innovation Fund (18CXZ011). We thank all medical staff and health practitioners who have contributed to the reporting of HFMD cases. 
Disclaimer: The funders had no role in the study design, data collection, data analysis, data interpretation, or writing of the report.

Disclosure: The corresponding authors had full access to all of the data in this study and had final responsibility for the decision to submit for publication. The authors declare that the research was conducted in the absence of any commercial or financial relationships that could be construed as a potential conflict of interest.

Authors' addresses: Tianci Guo, Junjiang Chen, Yong Long, Shuxuan Song, Zhongjun Shao, and Kun Liu, Department of Epidemiology, Ministry of Education Key Lab of Hazard Assessment and Control in Special Operational Environment, School of Public Health, Air Force Medical University, Xi'an, P.R. China, E-mails:714735719@qq.com, 861986602@ qq.com, longyong@fmmu.edu.cn, 897437763@qq.com, 13759981783@ 163.com, and liukun5959@qq.com. Yao Bai, Department of Epidemiology, Ministry of Education Key Lab of Hazard Assessment and Control in Special Operational Environment, School of Public Health, Air Force Medical University, Xi'an, P. R. China, and Department of Infectious Disease Control and Prevention, Xi'an Center for Disease Prevention and Control, Xi'an, P. R. China, E-mail: baiyaocdc@163.com. Jifeng Liu and Baozhong Chen, Department of Infectious Disease Control and Prevention, Xi'an Center for Disease Prevention and Control, Xi'an, P. R. China, E-mails: liujf19790113@163.com and cly19986023@sina.com.

This is an open-access article distributed under the terms of the Creative Commons Attribution (CC-BY) License, which permits unrestricted use, distribution, and reproduction in any medium, provided the original author and source are credited.

\section{REFERENCES}

1. Koh WM, Bogich T, Siegel K, Jin J, Chong EY, Tan CY, Chen MI, Horby P, Cook AR, 2016. The epidemiology of hand, foot and mouth disease in Asia: a systematic review and analysis. Pediatr Infect Dis J 35: 285-300.

2. Huang MC, Wang SM, Hsu YW, Lin HC, Chi CY, Liu CC, 2006. Long-term cognitive and motor deficits after enterovirus 71 brainstem encephalitis in children. Pediatrics 118: 1785-1788.

3. Chang LY, Huang LM, Gau SS, Wu YY, Hsia SH, Fan TY, Lin KL, Huang YC, Lu CY, Lin TY, 2007. Neurodevelopment and cognition in children after enterovirus 71 infection. $N$ Engl $J$ Med 356: 1226-1234.

4. Xing W et al., 2014. Hand, foot, and mouth disease in China, 2008-12: an epidemiological study. Lancet Infect Dis 14: 308-318.

5. Song JH, Park K, Shim A, Kwon BE, Ahn JH, Choi YJ, Kim JK, Yeo SG, Yoon K, Ko HJ, 2015. Complete sequence analysis and antiviral screening of medicinal plants for human coxsackievirus a16 isolated in Korea. Osong Public Health Res Perspect 6: $52-58$.

6. Ang LW, Koh BK, Chan KP, Chua LT, James L, Goh KT, 2009. Epidemiology and control of hand, foot and mouth disease in Singapore, 2001-2007. Ann Acad Med Singapore 106-112.

7. Nelson BR, Edinur HA, Abdullah MT, 2019. Compendium of hand, foot and mouth disease data in Malaysia from years 2010-2017. Data Brief 24: 103868.

8. Takahashi $\mathrm{S}$ et al., 2018. Epidemic dynamics, interactions and predictability of enteroviruses associated with hand, foot and mouth disease in Japan. J R Soc Interface 15: 20180507.

9. World Health Organization, 2014. Hand, Foot, and Mouth Disease situation Update. Available at: http://www.wpro.who.int/emerging diseases/hfmd_biweekly_29dec2014. pdf?ua=1. Accessed December 3, 2019.

10. Yi EJ, Shin YJ, Kim JH, Kim TG, Chang SY, 2017. Enterovirus 71 infection and vaccines. Clin Exp Vaccine Res 6: 4-14.

11. Zhu Q, Hao Y, Ma J, Yu S, Wang Y, 2011. Surveillance of hand, foot, and mouth disease in mainland China (2008-009). Biomed Environ Sci 24: 349-356.

12. Chang LY et al., 2002. Risk factors of enterovirus 71 infection and associated hand, foot, and mouth disease/herpangina in children during an epidemic in Taiwan. Pediatrics 109: e88.

13. Chen C, Lin H, Li X, Lang L, Xiao X, Ding P, He P, Zhang Y, Wang M, Liu Q, 2014. Short-term effects of meteorological factors on children hand, foot and mouth disease in Guangzhou, China. Int J Biometeorol 58: 1605-1614.
14. Wang H, Du Z, Wang X, Liu Y, Yuan Z, Liu Y, Xue F, 2015. Detecting the association between meteorological factors and hand, foot, and mouth disease using spatial panel data models. Int J Infect Dis 34: 66-70.

15. Qi $\mathrm{H}$ et al., 2018. Impact of meteorological factors on the incidence of childhood hand, foot, and mouth disease (HFMD) analyzed by DLNMs-based time series approach. Infect Dis Poverty 7: 7.

16. Zhao Q et al., 2018. Modeling the present and future incidence of pediatric hand, foot, and mouth disease associated with ambient temperature in mainland China. Environ Health Perspect 126: 047010.

17. Xu C, 2017. Spatio-temporal pattern and risk factor analysis of hand, foot and mouth disease associated with under-five morbidity in the Beijing-Tianjin-Hebei region of China. Int $J$ Environ Res Public Health 14: E416.

18. Tian L, Liang F, Xu M, Jia L, Pan H, Clments ACA, 2018. Spatiotemporal analysis of the relationship between meteorological factors and handfoot-mouth disease in Beijing, China. BMC Infect Dis 18: 158.

19. Yin F, Zhao T, Liu L, Lv Q, Li X, 2016. The association between ambient temperature and childhood hand, foot, and mouth disease in Chengdu, China: a distributed lag non-linear analysis. Sci Rep 6: 27305.

20. Wu J, Cheng J, Xu Z, Zhao K, Zhao D, Xie M, Yang H, Wen L, Li K, $\mathrm{Su} H, 2016$. Nonlinear and interactive effects of temperature and humidity on childhood hand, foot and mouth disease in Hefei, China. Pediatr Infect Dis J 35: 1086-1091.

21. Liu J et al., 2019. Epidemic pattern of hand-foot-and-mouth disease in Xi'an, China from 2008 through 2015. BMC Infect Dis 19: 19.

22. National Health Commission of the People's Republic of China, 2013. Law of the People's Republic of China on the Prevention and Treatment of Infectious Diseases. Available at: http://www.nhc.gov.cn/fzs/ s3576/201808/6d00c158844f42c5bc4993bffa665a.shtml. Accessed December 3, 2019.

23. National Health Commission of the People's Republic of China, 2018. Guidelines for Diagnosis and Treatment of Hand Foot Mouth Disease. Available at: http://www.nhc.gov.cn/xxgk/. Accessed December 3, 2019.

24. Gasparrini A, Armstrong B, Kenward MG, 2010. Distributed lag non-linear models. Stat Med 29: 2224-2234.

25. Zhang Y, Wei Z, Li G, 2017. Epidemiological analysis of handfoot-mouth disease in Beijing, 2007-2015. Mod Prev Med 44: 2689-2699.

26. Guo J et al., 2016. Analysis on epidemiological characteristics of hand-foot-mouth disease. Prev Med Trib 22: 433-435.

27. Chen QD, Sun M, 2015. Epidemiology of hand foot and mouth disease in permission of use these data from Xi'an CDC. Tianjin, 2013-2014. Dis Surveil 30: 463-467.

28. Qi Y, An XD, Chen Y, Wang B, Chen HJ, 2018. Analysis on epidemiological characteristics of hand-foot-mouth disease, Shenyang city, 2012-2016. Prev Med Trib 24: 35-38.

29. Zheng $Y$ et al., 2015. Epidemiological and etiological characteristics of hand-foot-mouth disease in Shaanxi Province. 2009 -2013. Chin J Dis Contro Z Pre 19: 135-137.

30. Kim YM, Park JW, Cheong HK, 2012. Estimated effect of climatic variables on the transmission of Plasmodium vivax malaria in the Republic of Korea. Environ Health Perspect 120: 1314-1319.

31. Xiao Y, He L, Chen Y, Wang Q, Meng Q, Chang W, 2018. The influence of meteorological factors on tuberculosis incidence in southwest China from 2006 to 2015. Sci Rep 8: 10053.

32. Hu WB, Li Y, Han W, Xue L, Zhang W, Ma W, Bi P, 2018. Meteorological factors and the incidence of mumps in Fujian province, China, 2005-2013: non-linear effects. Sci Total Environ 619-620: 1286-1298.

33. Hagiwara A, Yoneyama T, Hashimoto I, 1983. Isolation of a temperature-sensitive strain of enterovirus 71 with reduced neurovirulence for monkeys. J Gen Virol 64: 499-502.

34. Edwards NM, Myer GD, Kalkwarf HJ, Woo JG, Khoury PR, Hewett TE, Daniels SR, 2015. Outdoor temperature, precipitation, and wind speed affect physical activity levels in children: a longitudinal cohort study. J Phys Act Health 12: 1074-1081.

35. Yeager JG, O'Brien RT, 1979. Enterovirus inactivation in soil. Appl Environ Microbiol 38: 694-701. 
36. Averett N, 2016. Pacific island countries and climate change: examining associated human health vulnerabilities. Environ Health Perspect 124: A208.

37. Hess JJ, Saha S, Luber G, 2014. Summertime acute heat illness in U.S. emergency departments from 2006 through 2010: analysis of a nationally representative sample. Environ Health Perspect 122: $1209-1215$.

38. Zhang WJ, Zhang DM, Yu SC, Hao YT, 2016. Boosted regression tree model-based assessment of the impacts of meteorological drivers of hand, foot and mouth disease in Guangdong, China. Sci Total Environ 553: 366-371.

39. Zhao Y, Richardson B, Takle E, Chai L, Schmitt D, Xin H, 2019. Airborne transmission may have played a role in the spread of 2015 highly pathogenic avian influenza outbreaks in the United States. Sci Rep 9: 11755.

40. Xu Z, Etzel RA, Su H, Huang C, Guo Y, Tong S, 2012. Impact of ambient temperature on children's health: a systematic review. Environ Res 117: 120-131.

41. Cheng J, Wu J, Xu Z, Zhu R, Wang X, Li K, Wen L, Yang H, Su H, 2014. Associations between extreme precipitation and childhood hand, foot and mouth disease in urban and rural areas in Hefei, China. Sci Total Environ 497-498: 484-490.
42. Wang $Y$ et al., 2011. Hand, foot, and mouth disease in China: patterns of spread and transmissibility. Epidemiology 22: 781-792.

43. Liu W et al., 2015. Spatiotemporal dynamics of hand-foot-mouth disease and its relationship with meteorological factors in Jiangsu province, China. PLoS One 10: e0131311.

44. Phung D, Nguyen HX, Nguyen HLT, Do CM, Tran QD, Chu C, 2018. Spatiotemporal variation of hand-foot-mouth disease in relation to socioecological factors: a multiple-province analysis in Vietnam. Sci Total Environ 610-611: 983-991.

45. Ooi EE, Phoon MC, Ishak B, Chan SH, 2002. Seroepidemiology of human enterovirus 71, Singapore. Emerging Infect Dis 8: 995-997.

46. Hsu BM, Chen $\mathrm{CH}$, Wan MT, 2007. Genetic diversity of epidemic enterovirus 71 strains recovered from clinical and environmental samples in Taiwan. Virus Res 126: 69-75.

47. Zhang Y, Cui C, Shi L, 2015. Analysis on epidemiological characteristics of hand-foot-mouth disease in Xi'an, 2010-2013. Mod Prev Med 42: 1162-1164.

48. Xie Y, Xia L, Yuan J, 2019. Investigation on inapparent infection status of hand-foot-mouth disease in Xi'an city of Shaanxi province. J Clin Med Prac 23: 45-52. 